\title{
Data Plane Elements for Optical Performance Monitoring Agnostic to the Modulation Format for Disaggregated Optical Networks
}

\author{
Josep M. Fabrega ${ }^{1}$, Fabiano Locatelli ${ }^{1}$, Laia Nadal $^{1}$, Konstantinos Christodoloupoulos ${ }^{2}$, \\ Michela Svaluto Moreolo ${ }^{1}$, and Salvatore Spadaro ${ }^{3}$ \\ ${ }^{1}$ Centre Tecnològic de Telecomunicacions de Catalunya (CTTC/CERCA), Castelldefels (Spain) \\ ${ }^{2}$ Nokia Bell Labs Germany, Stuttgart (Germany) \\ ${ }^{3}$ Universitat Politècnica de Catalunya (UPC-BarcelonaTech), Barcelona (Spain) \\ e-mail: jmfabrega@cttc.es
}

\begin{abstract}
In this paper, data plane alternatives for optical performance monitoring are presented as enablers to address the key challenges in disaggregated optical networks. In fact, a key element of the disaggregated networks is optical performance monitoring that is expected to deliver the feedback needed by the control plane to guarantee end-to-end quality of transmission and quality of service. Therefore, we will discuss data plane elements for non-intrusive monitoring agnostic to the modulation format, proposing and analyzing different schemes. Furthermore, we will also review the relevant figures of merit to be delivered to the /SDN/ control, orchestration and management planes and their potential impact on the network performance.
\end{abstract}

Keywords Optical performance monitoring, disaggregated optical networks

\section{INTRODUCTION}

The approach of disaggregation of a chassis-based design into commodity (off-the-shelf) components has been gaining popularity, since it allows telecommunication operators and service providers to appropriately size their infrastructure and grow as needed [1], [2]. In fact, different models of disaggregation are proposed, ranging from a partial disaggregation to a full disaggregation model. In the first model, the transponders are provided by multiple vendors with open application programming interfaces (APIs) to interact with the transport softwaredefined networking (SDN) controller, whilst the remaining elements, known as optical line system (OLS), remain as a single-vendor infrastructure [2]. The OLS controller is provided by its vendor with open APIs to interface with the transport SDN controller. In the second model, all optical network elements can be provided by different vendors with standard APIs to the transport SDN controller. Each of these elements has a unified data modelling and open APIs to the SDN control system.

Proactive and reactive automation of optical networks when approaching the disaggregation paradigm in either of the models presented, is arguably the most significant challenge that needs to be addressed in order to further increase efficiency and cost-effectiveness of telecommunication networks. Such automation is based on actively processing network monitoring information and learning from the effects of the decisions taken to validate and provide optimal selection of network resources to satisfy the different service demands and dynamically re-optimize them when needed. One of the key elements of the network is the optical performance monitoring that is expected to deliver the feedback needed for guaranteeing end-to-end quality of transmission (QoT) and quality of service (QoS). Several techniques can be employed for acquiring the suitable figures of merit, ranging from highly intrusive receiver-based monitoring to non-intrusive optical probes independent from the modulation format [3], [4].

The QoT of a lightpath can be acquired at the transceivers by means of several figures of merit and techniques. Among the different available options for such transceivers, a convenient one is based on coherent reception [4]. This option can provide many interesting figures of merit, including bit error ratio (BER) prior to error correction, optical signal to noise ratio (OSNR), Q-factor, wavelength, power, chromatic dispersion, and relative state of polarization [4]. This allows the control and management plane to take the right decision at any time. Since optical disaggregated elements are particularly attractive for the metro and access network segments, that are particularly cost-sensitive, cost effective and high capacity solutions are pursued. For example, direct detection orthogonal frequency-division multiplexing (OFDM) transceivers are a promising solution, since they can provide high capacity at low cost while acquiring a wide range of figures of merit, including BER, signal to noise ratio (SNR) and power among others [5].

However, the aforementioned technologies are highly intrusive, since they require to actually demodulate and eventually equalize the signals in order to obtain the proposed figures of merit. Furthermore, they can only act channel by channel and path by path. So to have a picture of the occupancy and performance of a certain link/node is quite challenging, also posing difficulties for a complete diagnose of the network status. In order to solve this issue, a non-intrusive optical performance monitoring system can be deployed at the network nodes to automatically extract the different performance parameters. 
In this paper we advance the network monitoring probes discussed in [6], which are based on optical spectrum analysis. In fact, their modular approach comes with different options for optoelectronic front-ends and digital signal processing (DSP) modules [6], [7], allowing the measurements to be enhanced by using artificial intelligence [8]. Precisely, now we present an agile optoelectronic front-end scheme that is able to deliver arbitrary spectral resolution.

\section{CONCEPT AND ANALYSIS}

Figure 1(a) shows the basic concept of the monitoring probes, as described in [6]. It is an entire modular approach where two main blocks are identified: the optical spectrum analyzer (OSA) and a suitable monitoring agent able to extract different parameters and figures of merit from the optical spectrum while providing a communication interface with the control plane.
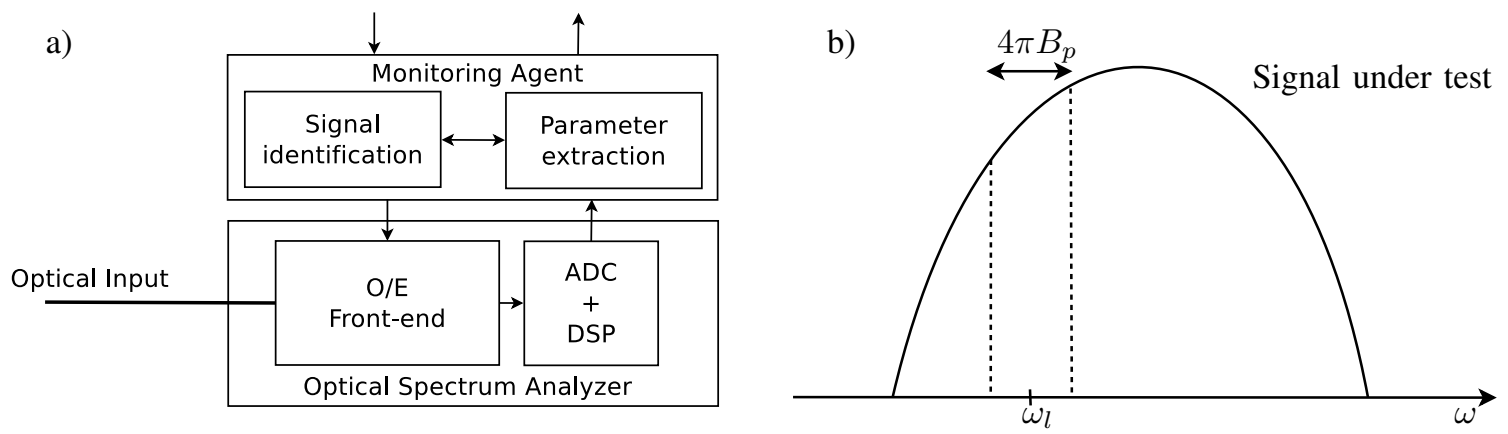

Figure 1. (a) Generic scheme for the monitoring probes [6]. (b) Signal acquisition.

The OSA can be regarded as an optical/electrical (O/E) front-end plus an analog to digital converter (ADC) and an eventual DSP. So, it can be controlled by the monitoring agent by means of an agreed API or by low-level commands interfacing the different devices composing the OSA in case a full custom design is approached.

In this paper we approach a custom OSA design based on coherent detection, whose scheme is shown in Fig. 2. There we can observe that there is a simple coherent detector featuring polarization diversity [9] and a complex DSP after signal digitization. This scheme is used to detect different spectral slices of the signal under test, calculate the optical spectrum of each of these slices and further reconstruct the entire optical spectrum by putting together the spectrum of each slice. So, using the local laser (oscillating at a frequency $\omega_{l}$ ), we tune the coherent detector to the desired frequency in order to detect a single slice of the signal under test. Therefore, we are able to detect the signal that lies within the range $\omega_{l} \pm 2 \pi B_{p}$, being $B_{P}$ the bandwidth of the photodiodes of the coherent detector. The detected signal slice is then processed and the spectrum reconstructed accordingly. Therefore, we obtain a polarization-resolved optical spectrum measurement. In the upcoming paragraphs we provide a formal description of the DSP in order to demonstrate how the signal slices are reconstructed.

Going to the details, we can represent the slice of the signal under test by the corresponding Jones vector

$$
E_{r}(t)=\left[\begin{array}{c}
\sqrt{P_{r}(t)} \cos \psi_{r} \exp \left(j \omega_{r} t+j \phi_{r}(t)\right) \\
\sqrt{P_{r}(t)} \sin \psi_{r} \exp \left(j \omega_{r} t+j \phi_{r}(t)+\theta_{r}\right)
\end{array}\right]
$$

being $P_{r}(t)$ its power, $\omega_{r}$ the angular frequency, $\phi_{r}(t)$ a generic phase accounting for phase noise and others, $\psi_{r}$ the polarization azimuth, and $\theta_{r}$ the polarization ellipticity.

Similarly, the local oscillator signal can be written as

$$
E_{l}(t)=\left[\begin{array}{c}
\sqrt{P_{l}} \cos \psi_{l} \exp \left(j \omega_{l} t+j \phi_{l}(t)\right) \\
\sqrt{P_{l}} \sin \psi_{l} \exp \left(j \omega_{l} t+j \phi_{l}(t)+\theta_{l}\right)
\end{array}\right] \simeq\left[\begin{array}{c}
\sqrt{\frac{P_{l}}{2}} \exp \left(j \omega_{l} t+j \phi_{l}(t)\right) \\
\sqrt{\frac{P_{l}}{2}} \exp \left(j \omega_{l} t+j \phi_{l}(t)\right)
\end{array}\right]
$$

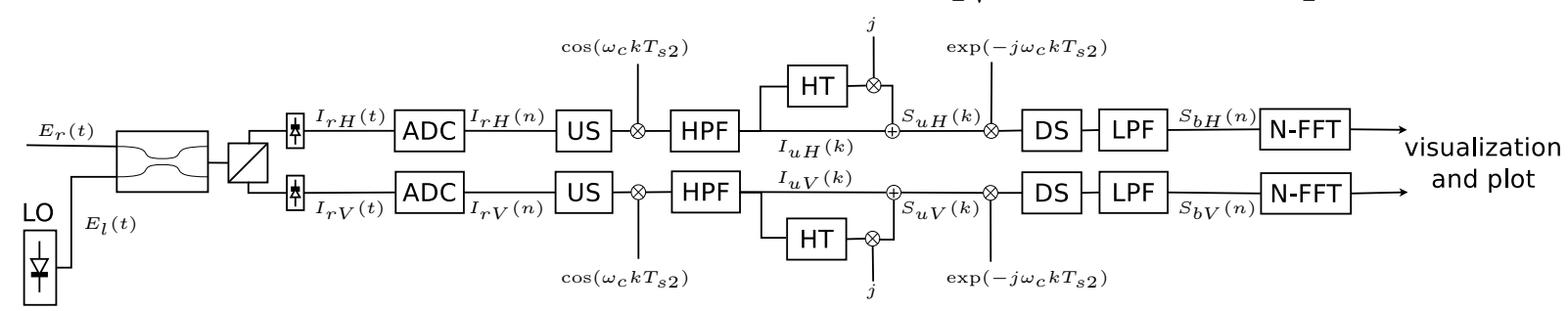

Figure 2. Scheme of the proposed front-end. DS: downsampling; HPF: high-pass filter; HT: Hilbert transform; LPF: low-pass filter; US: upsampling. 
where $P_{l}$ is its power, $\omega_{l}$ is the angular frequency, $\phi_{l}(t)$ is also a generic phase accounting for phase noise and others, $\psi_{l}$ is the azimuth of the state of polarization, and $\theta_{l}$ is the polarization ellipticity. Taking the polarization beam splitter (PBS) as reference for the state of polarization, we can assume that the local oscillator is featuring $\psi_{l}=45^{\circ}$ and $\theta_{l} \simeq 0$. In that case, both components of the Jones vector are equal.

At the photodetectors, the output currents can be expressed as

$$
\begin{aligned}
I_{r H}(t) & =\frac{R}{2}\left((1-\alpha) P_{r}(t) \cos ^{2} \psi_{r}+\alpha P_{l} / 2\right)+R \sqrt{\frac{\alpha(1-\alpha) P_{r}(t) P_{l}}{2}} \cos \psi_{r} \cos \left(\Delta \omega t+\phi_{e}(t)\right) \\
I_{r V}(t) & =\frac{R}{2}\left((1-\alpha) P_{r}(t) \sin ^{2} \psi_{r}+\alpha P_{l} / 2\right)+R \sqrt{\frac{\alpha(1-\alpha) P_{r}(t) P_{l}}{2}} \sin \psi_{r} \cos \left(\Delta \omega t+\phi_{e}(t)+\theta_{r}\right)
\end{aligned}
$$

where $R$ is the photodiode responsivity, $\alpha$ is the coupling ratio, $\Delta \omega=\omega_{r}-\omega_{l}$, and $\phi_{e}(t)=\phi_{r}(t)-\phi_{l}(t)$. These equations can be further simplified for $\alpha=1 / 2$ (i.e. using a 50:50 coupler) and assuming that the coherent term is much higher that the direct detection terms. In that case

$$
\begin{aligned}
& I_{r H}(t)=R \sqrt{\frac{P_{r}(t) P_{l}}{8}} \cos \psi_{r} \cos \left(\Delta \omega t+\phi_{e}(t)\right) \\
& I_{r V}(t)=R \sqrt{\frac{P_{r}(t) P_{l}}{8}} \sin \psi_{r} \cos \left(\Delta \omega t+\phi_{e}(t)+\theta_{r}\right)
\end{aligned}
$$

So, we obtain the real part of the slice examined from signal under test, including the information on its state of polarization. Also, this pair of signals is limited in bandwidth to the bandwidth of the photodiodes $B_{p}$. $I_{r H}$ and $I_{r V}$ are then digitized and stored in an array of $N$ samples

$$
\begin{aligned}
& I_{r H}(n)=R \sqrt{\frac{P_{r}\left(n T_{s}\right) P_{l}}{8}} \cos \psi_{r} \cos \left(\Delta \omega n T_{s}+\phi_{e}\left(n T_{s}\right)\right) \\
& I_{r V}(n)=R \sqrt{\frac{P_{r}\left(n T_{s}\right) P_{l}}{8}} \sin \psi_{r} \cos \left(\Delta \omega n T_{s}+\phi_{e}\left(n T_{s}\right)+\theta_{r}\right)
\end{aligned}
$$

being $T_{s}=1 / f_{s}$ the sampling period, $f_{s}$ the sampling frequency, and $1 \leq n \leq N$.

This is then upsampled to a frequency $f_{s 2}$, much higher than $f_{s}$, so that the signals $I_{r H}(n)$ and $I_{r V}(n)$ can be upconverted to $\omega_{c} \gg 2 \pi B_{p}$ and high-pass filtered to obtain

$$
\begin{aligned}
& I_{u H}(k)=R \sqrt{\frac{P_{r}\left(k T_{s 2}\right) P_{l}}{32}} \cos \psi_{r} \cos \left(\left(\Delta \omega+\omega_{c}\right) k T_{s 2}+\phi_{e}\left(k T_{s 2}\right)\right) \\
& I_{u V}(k)=R \sqrt{\frac{P_{r}\left(k T_{s 2}\right) P_{l}}{32}} \sin \psi_{r} \cos \left(\left(\Delta \omega+\omega_{c}\right) k T_{s 2}+\phi_{e}\left(k T_{s 2}\right)+\theta_{r}\right)
\end{aligned}
$$

where $T_{s 2}=1 / f_{s 2}$ is the sampling period, $M$ is the number of samples of the array, $M / N$ is the upsampling ratio, and $1 \leq k \leq M$.

Therefore, the Bedrosian theorem is applicable and we can obtain the corresponding imaginary part of each detected signal by applying the Hilbert transform [10]. So, the corresponding complex signal can be reconstructed

$$
\begin{aligned}
& S_{u H}(k)=R \sqrt{\frac{P_{r}\left(k T_{s 2}\right) P_{l}}{32}} \cos \psi_{r} \exp \left(j\left(\Delta \omega+\omega_{c}\right) k T_{s 2}+j \phi_{e}\left(k T_{s 2}\right)\right) \\
& S_{u V}(k)=R \sqrt{\frac{P_{r}\left(k T_{s 2}\right) P_{l}}{32}} \sin \psi_{r} \exp \left(j\left(\Delta \omega+\omega_{c}\right) k T_{s 2}+j \phi_{e}\left(k T_{s 2}\right)+j \theta_{r}\right)
\end{aligned}
$$

This signal is then downconverted, downsampled and low-pass filtered to obtain a baseband representation

$$
\begin{aligned}
& S_{b H}(n)=R \sqrt{\frac{P_{r}\left(n T_{s}\right) P_{l}}{32}} \cos \psi_{r} \exp \left(j \Delta \omega n T_{s}+j \phi_{e}\left(n T_{s}\right)\right) \\
& S_{b V}(n)=R \sqrt{\frac{P_{r}\left(n T_{s}\right) P_{l}}{32}} \sin \psi_{r} \exp \left(j \Delta \omega n T_{s}+j \phi_{e}\left(n T_{s}\right)+j \theta_{r}\right)
\end{aligned}
$$

$S_{b H}(n)$ and $S_{b V}(n)$ are a baseband representation of the detected slice of the signal under test, with a light dependence on the frequency difference $\Delta \omega$ and the phase difference $\phi_{e}\left(n T_{s}\right)$ between the local laser and this signal slice. So, applying the $N$-point fast Fourier transform (FFT) we obtain the desired slice of the optical spectrum. 


\section{DISCUSSION AND CONCLUSIONS}

From the mathematical expressions obtained, we can derive a set of requirements and tradeoffs for the proposed technique. As stated before, the photodetectors limit the bandwidth that can be acquired at each step tuning the local oscillator. So, the steps taken by the local oscillator have to be at maximum $4 \pi B_{p}$. In case we want to tune the local laser in very coarse steps, the bandwidth of the photodiodes should be as large as possible.

Nevertheless, the bandwidth of the photodetectors also sets a bound for the sampling frequency of the ADCs. In order to avoid any aliasing, $f_{s}$ should be greater than $2 B_{p}$. In turn, $f_{s}$ also limits the frequency resolution that can be obtained after the FFT, which is $f_{s} / N$.

As previously discussed, the reconstructed signal slice after coherent detection features a dependency on the phase and frequency difference between the local laser and the signal slice. Even this is not discussed in this paper, phase and frequency estimation techniques should be applicable in order to minimize this difference.

Regarding the wavelength range covered by the proposed optoelectronic front-end, it is limited by its optical components, mainly by the local laser. So, a broadly tunable laser is desired to cover a large wavelength range.

Summarizing, in this paper we have presented a method for estimating the optical spectrum at high resolution using a rather simple front-end based on coherent detection. This technique relies on detecting different slices of the optical spectrum. So, there is no need for continuous frequency sweep as in the so-called coherent optical spectrum analyzer [11]. In our case, we can use a tunable laser using eventually coarse discrete steps, depending on the bandwidth of the photodiodes.

This constitutes an advancement in optical performance monitoring techniques in order to create a common subsystem that is agnostic to the optical signal waveforms (which may feature different multiplexing schemes and modulation formats) and capable to provide the suitable figures of merit to the control, orchestration and management planes.

\section{ACKNOWLEDGMENT}

This work was supported by the Spanish MINECO project AURORAS (RTI2018-099178-B-I00) and EU-H2020 project ONFIRE (G.A. 765275).

\section{REFERENCES}

[1] M. D. Leenheer, T. Tofigh, and G. Parulkar, "Open and Programmable Metro Networks," in Optical Fiber Communication Conference. Optical Society of America, 2016, p. Th1A.7.

[2] E. Riccardi, P. Gunning, O. G. de Dios, M. Quagliotti, V. Lopez, and A. Lord, "An Operator view on the Introduction of White Boxes into Optical Networks," Journal of Lightwave Technology, vol. 36, no. 15, pp. 3062-3072, 2018.

[3] C. C. K. Chan, Ed., Optical performance monitoring: advanced techniques for next-generation photonic networks. Burlington, MA: Academic Press/Elsevier, 2010, oCLC: ocn470818731.

[4] A. P. Vela, M. Ruiz, F. Fresi, N. Sambo, F. Cugini, G. Meloni, L. Potì, L. Velasco, and P. Castoldi, "BER Degradation Detection and Failure Identification in Elastic Optical Networks," Journal of Lightwave Technology, vol. 35, no. 21, pp. 4595-4604, Nov 2017.

[5] M. Svaluto Moreolo, J. M. Fabrega, L. Nadal, F. J. Vílchez, A. Mayoral, R. Vilalta, R. Munoz, R. Casellas, R. Martínez, M. Nishihara, T. Tanaka, T. Takahara, J. C. Rasmussen, C. Kottke, M. Schlosser, R. Freund, F. Meng, S. Yan, G. Zervas, D. Simeonidou, Y. Yoshida, and K.-I. Kitayama, "SDN-Enabled Sliceable BVT Based on Multicarrier Technology for Multiflow Rate/Distance and Grid Adaptation," J. Lightwave Technol., vol. 34, no. 6, pp. 1516-1522, Mar 2016.

[6] J. M. Fàbrega, M. Svaluto Moreolo, and L. Nadal, "Optical performance monitoring systems in disaggregated optical networks," in 2018 20th International Conference on Transparent Optical Networks (ICTON), 2018, pp. 1-4.

[7] B. Shariati, M. Ruiz, J. Comellas, and L. Velasco, "Learning from the optical spectrum: Failure detection and identification," J. Lightwave Technol., vol. 37, no. 2, pp. 433-440, Jan 2019. [Online]. Available: http://jlt.osa.org/abstract.cfm?URI=jlt-37-2-433

[8] F. Locatelli, K. Christodoulopoulos, M. S. Moreolo, J. M. Fàbrega, and S. Spadaro, "Machine LearningBased in-Band OSNR Estimation From Optical Spectra," IEEE Photonics Technology Letters, vol. 31, no. 24, pp. 1929-1932, 2019.

[9] J. A. Altabas, L. F. Suhr, G. S. Valdecasa, J. A. Lazaro, I. Garces, J. B. Jensen, and A. T. Clausen, "25gbps quasicoherent receiver for beyond ng-pon2 access networks," in 2018 European Conference on Optical Communication (ECOC), 2018, pp. 1-3.

[10] E. Bedrosian, "A product theorem for Hilbert transforms," Proceedings of the IEEE, vol. 51, no. 5, pp. 868-869, 1963.

[11] D. Baney, B. Szafraniec, and A. Motamedi, "Coherent Optical Spectrum Analyzer," Photonics Technology Letters, IEEE, vol. 14, no. 3, pp. 355-357, March 2002. 